\title{
Die thermische Entmischung von Gasen bei hohem Druck
}

\author{
Von ERwin WiLly Becker \\ Aus dem Physikalischen Institut der Universität Marburg (Lahn) \\ (Z. Naturforschg. 5 a, 457-465 [1950]; eingegangen am 5. Juli 1950)
}

\begin{abstract}
Es wird die Druckabhängigkeit des Thermodiffusionsfaktors $\alpha$ einiger Gaspaare durch Untersuchung der elementaren thermischen Entmischung bestimmt. Für etwa äquimolare Mischungen steigt $\alpha$ bei einer mittleren Temperatur von $84^{\circ} \mathrm{C}$ im Bereich von $3-80 \mathrm{~atm}$ bei $\mathrm{CO}_{2} / \mathrm{H}_{2}$ von 0,32 auf 0,98 , bei $\mathrm{CO}_{2} / \mathrm{N}_{2}$ von 0,05 auf 0,40 , bei $\mathrm{CO}_{2} / \mathrm{A}$ von 0,03 auf 0,25 , bei $\mathrm{CO}_{2} / \mathrm{CH}_{4}$ von 0,07 auf 0,37 und bei $\mathrm{N}_{2} / \mathrm{H}_{2}$ von 0,35 auf 0,44 . Die Gaspaare $\mathrm{N}_{2} / \mathrm{CH}_{4}$ und $\mathrm{N}_{2} / \mathrm{NH}_{3}$ zeigen dagegen negative Druckabhängigkeit, die bei dem letzten schon bei 1,8 atm zu einem Vorzeichenwechsel von $\alpha$ führt. Die Temperatur- und Konzentrationsabhängigkeit des Thermodiffusionsfaktors von $\mathrm{CO}_{2} / \mathrm{H}_{2}$ besitzen bei $47 \mathrm{~atm}$ umgekehrtes Vorzeichen wie bei $2 \mathrm{~atm}$. Es wird theoretisch gezeigt, $\mathrm{da} \beta$ ein erheblicher Anteil der Druckabhängigkeit der thermischen Entmischung auf der Realität der Gase beruht und daher im Prinzip mit der Zustandsgleichung beschrieben werden kann. Der Einfluß der Realität der Gase auf den Diffusionsthermoeffekt wird diskutiert.
\end{abstract}

$\mathrm{B}$ ringt man ein homogenes Gemisch von zwei Gasen in ein Temperaturgefälle, so beobachtet man an der heißen Wand gewöhnlich eine geringfügige Anreicherung der Komponente mit dem kleineren Molekulargewicht, während der schwerere Partner etwas bevorzugt an der kalten Wand anzutreffen ist. Diese unter dem Namen Thermodiffusion bekannte Erscheinung wurde 1911 von Enskog${ }^{1}$ auf Grund einer Lösung der Boltzmannschen Fundamentalgleichung für nicht-isotherme Systeme vorausgesagt. Die eingehende Theorie ist in der Enskogschen Dissertation ${ }^{2}$ enthalten. Im Jahre 1917 veröffentlichte $\mathrm{Ch}$ a p m a n ${ }^{3}$, unabhängig von Enskog, eine theoretische Arbeit über denselben Gegenstand. Im gleichen Jahr konnte Ch a p m a nusammen mit D o ots on die Thermodiffusion erstmalig experimentell nachweisen ${ }^{4}$. Die Thermodiffusion blieb lange Zeit ziemlich unbeachtet. Erst in den letzten Jahren hat sie wegen des Isotopen-

1 D. En sk og, Physik. Z. 12, 56, 533 [1911].

2 D. Enskog, Kinetische Theorie der Vorgänge in mäßig verdünnten Gasen, Diss. Uppsala 1917.

3 S. Ch a p m a n, Philos. Trans. Roy. Soc. London, Ser. A 217, 115 [1917].

4 S. Ch a pman u. F. W. D o ot s o n, Philos. Mag. J. Sci. 33, 248 [1917].

5 K. Clusius u. G. Dickel, Naturwiss. 26, 546 [1938].

6 Zusammenfassende Darstellungen u. a. S. C h a p $m$ a n, Proc. Roy. Soc. [London] Ser. A 177, 38 [1940]; R. C. Jones u. W. H. F u rry, Rev. mod. Physics 18, 151 [1946]; S. Chapman u. T. G. Cowling, The Mathematical Theory of Non-Uniform Gases, (The Cambridge University Press, New York, 1939 (dem Verf. nicht zugänglich); vgl. auch A. Eu cken, Lehrbuch der Chemischen Physik II 1 , 2. Auflage, Leipzig 1943.

trennverfahrens von $\mathrm{Clusius}$ und $\mathrm{Dickel^{5 }}$ größeres Interesse erregt.

Aus der Theorie der Thermodiffusion, wie sie von Enskog, Chapman, Jones ${ }^{6}$ u. a. entwickelt worden ist, geht hervor, daß die Größe der Entmischung bei vorgegebener Temperaturdifferenz vom Gasdruck nicht wesentlich stärker abhängen sollte als die Zähigkeit der Gasmischung. Die zur Prüfung dieser theoretischen Aussage von verschiedenen Autoren durchgeführten Experimente ${ }^{7}$ erstrecken sich nur auf

7 I. W. H. L u g g, Philos. Mag. J. Sci. 8, 1019 [1929]; T. L. I b bs, K. E. Grew u. A. H irst, Proc. physic. Soc. 41, 456 [1929]; N. G. S c h m a h l u. W. K n e pp e r, Z. Elektrochem. 42, 681 [1936]; K. E. Grew, Nature [London] 156, 267 [1945]; H. G. D r i c k a m e r, J. R. H of t o, J. Chem. Physics 17, 1165 [1949]; N. G. $\mathrm{S}$ ch m a l u. J. S ch e w e, Z. Elektrochem. 46, 203 [1940]. Die in der letzten Arbeit beobachtete Abnahme der thermischen Entmischung von $\mathrm{H}_{2} \mathrm{~S} / \mathrm{H}_{2}$ und $\mathrm{CO}_{2} / \mathrm{H}_{2}$ mit steigendem Druck ist auf eine druckabhängige Konvektionsbewegung in dem horizontal liegenden Versuchsgefäß zurückzuführen, beruht also nicht auf einer negativen Druckabhängigkeit des Thermodiffusionsfaktors, wie die Verf. annehmen. (Die „negative Druckabhängigkeit“ ließ sich auch unsererseits leicht durch Horizontallegen der in Abb. 1 der vorliegenden Arbeit dargestellten Apparatur reproduzieren.) Auf die Notwendigkeit, bei der Messung von Thermodiffusionsfaktoren (zur Vermeidung der Konvektion) den heißen Behälter über dem kalten anzuordnen, hat schon $\mathrm{S}$. $\mathrm{C} \mathrm{h}$ a $\mathrm{p} \mathrm{m}$ a $\mathrm{n}$ hingewiesen (Philos. Mag. J. Sci. 38, 186 [1919]. In der früheren Arbeit haben $\mathrm{S}$ ch m a hl u. Kn e p per (s. oben) eine schwache positive Druckabhängigkeit der thermischen Entmischung von $\mathrm{H}_{2} / \mathrm{H}_{2} \mathrm{~S}$ im Bereich von $240-801$ Torr beobachtet, die wahrscheinlich z. Tl. auf dem in der vorliegenden Arbeit behandelten Effekt beruht, die aber $\mathrm{Schmahl} u$. S chewe in ihrer späteren Veröffentlichung (s. oben) für eine Täuschung halten. 
einen Druckbereich von wenigen Atmosphären, so daß die ausnahmslos innerhalb der Fehlergrenze gefundene Druck-Unabhängigkeit nicht als sichere Bestätigung der Theorie angesehen werden konnte. Da uns im Rahmen unserer Hochdruck-Trennrohrversuche ${ }^{8}$ der Verlauf der thermischen Entmischung bei wesentlich höheren Drucken interessierte, haben wir den Untersuchungsbereich erheblich ausgedehnt (bis $80 \mathrm{~atm})$ und dabei festgestellt, daß die thermische Entmischung gewöhnlich wesentlich stärker vom Gasdruck abhängt, als man nach der Enskog-Chapmanschen Theorie erwarten sollte ${ }^{9}$.

Diese Diskrepanz war im Grunde nicht verwunderlich, da die Thermodiffusionstheorien das Gasgemisch insofern idealisieren, als sie den Molekülen bis auf die kurze Dauer eines „Zusammenstoßes“ jegliche Wechselwirkung abschreiben. Mathematisch kommt diese Vereinfachung durch die Verwendung der Boltzmannschen Fundamentalgleichung in die Rechnung hinein, die bekanntlich unter der erwähnten Voraussetzung abgeleitet wird ${ }^{10}$.

In der vorliegenden Arbeit wird zunächst ausführlicher über die Entmischungsversuche bei hohem Druck berichtet. Danach wird gezeigt, wie man einen wesentlichen Teil der bei hohem Druck auftretenden neuen Erscheinungen prinzipiell mit der Zustandsgleichung der Gase beschreiben kann. Auf diese Möglichkeit wurde schon früher hingewiesen ${ }^{9,11}$. R. H a a s e hat inzwischen auf Grund unserer Versuche die Erscheinungen der thermischen Entmischung bei hohem Druck in einer theoretischen Arbeit thermodynamischphänomenologisch behandelt ${ }^{12}$. Da das Verfahren bei der Zuordnung der phänomenologischen Konstanten $\mathrm{zu}$ den experimentell meßbaren Größen aber naturgemäß nicht völlig zwingend ist, halten wir die Wiedergabe unserer modellmäßigen, unmittelbar an die Zustandsgleichung anknüpfenden Methode nicht für überflüssig.

\section{Die Entmischungsversuche}

Die Untersuchung wurde mit der in Abb. 1 dargestellten Apparatur durchgeführt. Zwei Stahlzylinder A und B von je $140 \mathrm{~cm}^{3}$ Inhalt stehen über ein kurzes engeres Stahlrohr C in Verbindung. Während B mit einem Kühlwassermantel umgeben ist, kann A von außen elektrisch

8 E. W. B e cker, Z. Naturforschg. 2a, 441, 447 [1947].

9 Vorläufige Mitteilung: E. W. B e cker u. A. S chulzeff, Naturwiss. 35, 218 [1948]; vorgetragen auf der Tagung der Deutschen Physikalischen Gesellschaft in Clausthal-Zellerfeld am 11. Sept. 1948. Die gefundene Druckabhängigkeit des Thermodiffusionsfaktors gibt die Erklärung für die in 8 beobachtete Steigerung des Wirkungsgrades der Trennrohrentmischung. geheizt werden. Die Temperaturdifferenz zwischen A und B wird mit einem Thermoelement bestimmt. Zwei Ventile ermöglichen die Entnahme von Proben aus beiden Gefäßen.

Die Gase wurden in einer Bombe gemischt und über eines der Ventile in die heiße Apparatur eingelassen. Der Druck konnte an einem Metallfeinmeßmanometer abgelesen werden. Nach einer Zeit, die für die Einstellung des stationären Zustandes sicher ausreichte ${ }^{13}$, wurden aus beiden Gefäßen Gasproben entnommen und chemisch oder mit der Wärmeleitfähigkeitsmethode analysiert ${ }^{14}$. Die Analysengenauigkeit betrug in beiden Fällen etwa

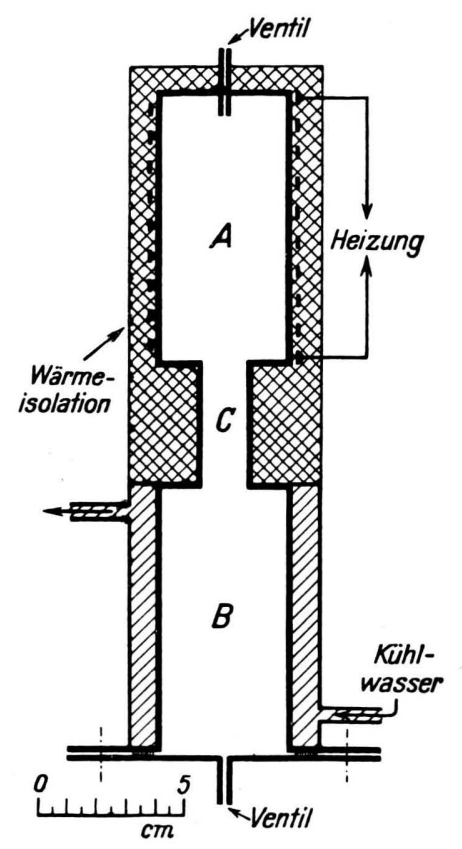

Abb. 1. Apparatur zur Untersuchung der thermischen Entmischung von Gasen bei hohem Druck.

$\pm 0,1 \%$. Um sicher zu wissen, wann der stationäre Endzustand vorlag, wurden mehrere Messungen zu verschiedenen Zeitpunkten angestellt.

Zunächst wurde die Druckabhängigkeit der thermischen Entmischung der Gaspaare $\mathrm{CO}_{2} / \mathrm{H}_{2}$, $\mathrm{CO}_{2} / \mathrm{N}_{2}, \mathrm{CO}_{2} / \mathrm{A}, \mathrm{CO}_{2} / \mathrm{CH}_{4}, \mathrm{~N}_{2} / \mathrm{CH}_{4}$ und $\mathrm{N}_{2} / \mathrm{H}_{2}$ untersucht. Die Temperaturdifferenz betrug in allen Fällen $140^{\circ}$, wobei die tiefere Temperatur durch die des Kühlwassers gegeben war $\left(\approx 14^{\circ} \mathrm{C}\right)$. Die Ergebnisse sind mit allen interessierenden Daten in der Tab. 1

10 L. B o ltz m a n n, Vorlesungen über Gastheorie I, 2. unverb. Aufl. S. 98, Leipzig 1910.

11 E. W. B e c k e r, Habil.-Schrift, Marburg, Jan. 1949.

12 R. H a a s e, Z. Physik 127, 1 [1950].

13 Die Relaxationszeit liegt, je nach dem Druck, in der Größenordnung von Stunden bis Tagen.

14 Für die Durchführung der Wärmeleitfähigkeitsanalysen danke ich Hrn. cand. phys. O. S t e h l. 


\begin{tabular}{|c|c|c|c|c|}
\hline Gaspaar & $\begin{array}{l}\text { Druck } \\
\text { (atm) }\end{array}$ & $\bar{\gamma}$ & $\Delta \gamma$ & $\alpha$ \\
\hline $\mathrm{CO}_{2} / \mathrm{H}_{2}$ & $\begin{array}{r}3 \\
26 \\
51 \\
81\end{array}$ & $\begin{array}{l}0,52 \\
0,52 \\
0,52 \\
0,52\end{array}$ & $\begin{array}{l}0,031_{5} \\
0,045_{5} \\
0,066_{5} \\
0,097_{0}\end{array}$ & $\begin{array}{l}0,31_{\odot} \\
0,46_{\circ} \\
0,67_{2} \\
0,98_{0}\end{array}$ \\
\hline $\mathrm{CO}_{2} / \mathrm{N}_{2}$ & $\begin{array}{r}3 \\
26 \\
52 \\
81\end{array}$ & $\begin{array}{l}0,48 \\
0,48 \\
0,48 \\
0,48\end{array}$ & $\begin{array}{l}0,005_{0} \\
0,012_{3} \\
0,025_{0} \\
0,039_{8}\end{array}$ & $\begin{array}{l}0,05_{0} \\
0,12_{4} \\
0,25_{2} \\
0,40_{2}\end{array}$ \\
\hline $\mathrm{CO}_{2} / \mathrm{A}$ & $\begin{array}{r}3 \\
26 \\
50 \\
80\end{array}$ & $\begin{array}{l}0,46 \\
0,46 \\
0,46 \\
0,46\end{array}$ & $\begin{array}{l}0,003_{0} \\
0,008_{2} \\
0,014_{8} \\
0,024_{6}\end{array}$ & $\begin{array}{l}0,03_{0} \\
0,08_{3} \\
0,15_{0} \\
0,24_{8}\end{array}$ \\
\hline $\mathrm{CO}_{2} / \mathrm{CH}_{4}$ & $\begin{array}{r}3 \\
26 \\
51 \\
81\end{array}$ & $\begin{array}{l}0,48 \\
0,48 \\
0,48 \\
0,48\end{array}$ & $\begin{array}{l}0,007_{1} \\
0,012_{0} \\
0,021_{5} \\
0,036_{2}\end{array}$ & $\begin{array}{l}0,07_{2} \\
0,12_{1} \\
0,21_{7} \\
0,36_{5}\end{array}$ \\
\hline $\mathrm{N}_{2} / \mathrm{CH}_{4}$ & $\begin{array}{r}4 \\
20 \\
38 \\
80\end{array}$ & $\begin{array}{l}0,50 \\
0,50 \\
0,50 \\
0,50\end{array}$ & $\begin{array}{l}0,007 \\
0,003 \\
0,002 \\
0,000\end{array}$ & $\begin{array}{l}0,07 \\
0,03 \\
0,02 \\
0,00\end{array}$ \\
\hline $\mathrm{N}_{2} / \mathrm{H}_{2}$ & $\begin{array}{r}3 \\
26 \\
50 \\
78\end{array}$ & $\begin{array}{l}0,50 \\
0,50 \\
0,50 \\
0,50\end{array}$ & $\begin{array}{l}0,034_{5} \\
0,036_{0} \\
0,039_{0} \\
0,043_{8}\end{array}$ & $\begin{array}{l}0,34_{8} \\
0,36_{4} \\
0,39_{4} \\
0,44_{2}\end{array}$ \\
\hline
\end{tabular}

Tab. 1. Abhängigkeit der thermischen Entmischung einiger Gaspaare vom Gasdruck. $T=287^{\circ}, T^{\prime}=427^{\circ}$ abs. $\bar{\gamma}=$ mittlerer Molenbruch der schweren Komponente. $\Delta \gamma=$ Differenz der Molenbrüche einer Komponente in den verschieden temperierten Gefäßen. $\alpha=$ Thermodiffusionsfaktor, berechnet mit Gl. (1).

zusammengestellt ${ }^{15}$. Die letzte Spalte enthält den Thermodiffusionsfaktor $\alpha$, der auf Grund der phänomenologischen Beziehung

$$
\Delta \gamma=\bar{\gamma}(1-\bar{\gamma}) \alpha \ln T^{\prime} / T
$$

aus der Entmischung $\Delta \gamma$ und den absoluten Temperaturen $T^{\prime}$ und $T$ von heißer und kalter Wand berechnet wurde. In Abb. 2 ist $\alpha$ in Abhängigkeit vom Gasdruck graphisch dargestellt. Man erkennt, daß die von der Enskog-Chapmanschen Theorie geforderte Druck-Unabhängigkeit in den meisten Fällen auch nicht annähernd erfüllt ist. Im Bereich von 3-80 atm nimmt $\alpha$ bei $\mathrm{CO}_{2} / \mathrm{H}_{2}$ um den Faktor 3 , bei $\mathrm{CO}_{2} / \mathrm{N}_{2}$ und $\mathrm{CO}_{2} / \mathrm{A}$ um den Faktor 8 und bei $\mathrm{CO}_{2} / \mathrm{CH}_{4}$ um

15 Die in ${ }^{9}$ mitgeteilten Zahlen weichen z. Tl. etwas von den Werten der Tab. 1 ab. Dies hat seinen Grund darin, daß die neueren Ergebnisse unter etwas anderen Temperaturverhältnissen gewonnen wurden und bei den älteren, mehr orientierenden Messungen, auf die Einhaltung einer bestimmten mittleren Konzentration weniger Wert gelegt wurde.

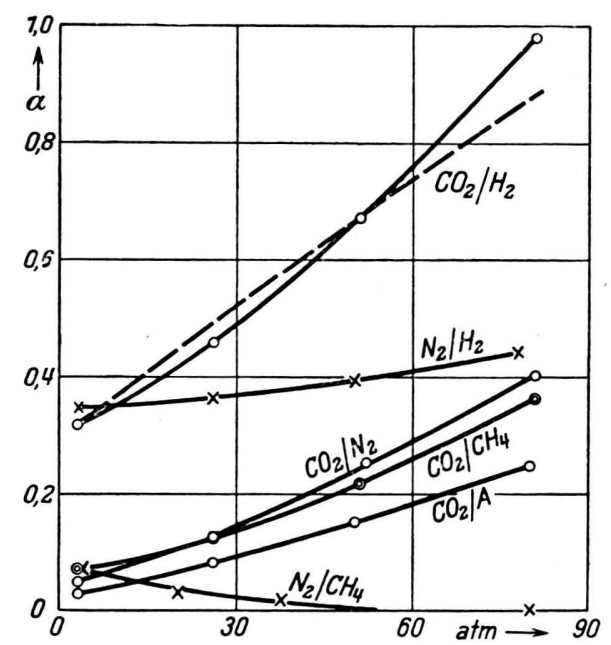

Abb. 2. Druckabhängigkeit des Thermodiffusionsfaktors $\alpha$ einiger Gaspaare. Für $\mathrm{CO}_{2} / \mathrm{H}_{2}$ ist der nach Gl. (19) und (20) zu erwartende Verlauf gestrichelt eingezeichnet.

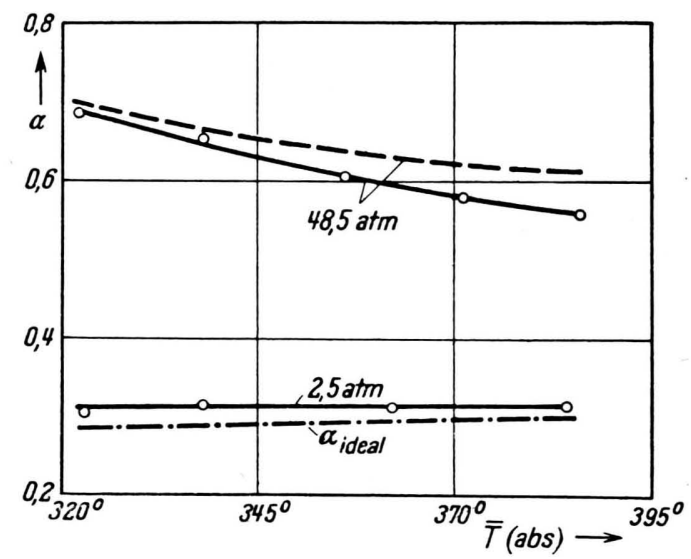

Abb. 3. Temperaturabhängigkeit des Thermodiffusionsfaktors $\alpha$ von $\mathrm{CO}_{2} / \mathrm{H}_{2}$ bei verschiedenen Drucken. $\bar{\gamma} \mathrm{CO}_{2}$ $=0,48$. Experimentelle Kurve ausgezogen. Gestrichelt: Der Verlauf bei 48,5 atm nach Gl.(19) und (20). Strichpunktiert: Der Verlauf von $\alpha_{\text {ideal }}$, berechnet mit (19) und (20) aus dem bei $2,5 \mathrm{~atm}$ gemessenen Verlauf von $\alpha$.

$$
\bar{T}=\frac{1}{2}\left(T^{\prime}+T\right) \text {. }
$$

den Faktor 5 zu. Bei $\mathrm{N}_{2} / \mathrm{H}_{2}$ beträgt die Zunahme dagegen nur etwa $30 \%$, während bei $\mathrm{N}_{2} / \mathrm{CH}_{4}$ sogar eine negative Druckabhängigkeit zu verzeichnen ist.

In einer zweiten Versuchsreihe wurde die $\mathrm{T}$ e $\mathrm{m}$ peraturabhängigkeit der thermischen Entmischung von $\mathrm{CO}_{2} / \mathrm{H}_{2}$ bei 2,5 und $48,5 \mathrm{~atm}$ bestimmt. Das Ergebnis ist in der Abb. 3 wiedergegeben. Während sich $\alpha$ bei $2,5 \mathrm{~atm}$ als praktisch unabhängig von der Temperatur erweist, nimmt der Thermodiffusionsfaktor bei 48,5 atm stark mit steigender mittlerer Temperatur $a b$. 
Schließlich wurde noch die Konzentrations a b hä ng i g k e it der thermischen Entmischung von $\mathrm{CO}_{2} / \mathrm{H}_{2}$ bei verschiedenen Drucken untersucht. Abb. 4 zeigt, daß der Thermodiffusionsfaktor bei 2 atm mit zunehmender Wasserstoffkonzentration stark ansteigt (in Übereinstimmung mit der Enskog-Chapmanschen Theorie). Bei $47 \mathrm{~atm}$ beobachtet man dagegen für die Konzentrationsabhängigkeit das umgekehrte Vorzeichen.



Abb. 4. Konzentrationsabhängigkeit des Thermodiffusionsfaktors $\alpha$ bei verschiedenen Drucken. Mittlere Temperatur $\bar{T}=90^{\circ} \mathrm{C}$. Experimentelle Kurve ausgezogen. Gestrichelt: Der Verlauf bei 47 und 26 atm nach Gl. (19) und (20). Strichpunktiert: Der Verlauf von $\alpha_{\text {ideal }}$, berechnet mit (19) und (20) aus dem bei 2,0 atm gemessenen Verlauf von $\alpha$.

\section{Abgrenzung der Thermodiffusion} quasi-idealer Gase

Die Enskog-Chapmansche Thermodiffusion der "quasi-idealen“ Gase ergibt sich als gaskinetischer Effekt zweiter Ordnung, wenn man bei der Aufstellung der Diffusionsgleichung eines im Temperaturgefälle befindlichen Gasgemisches die Störung der Geschwindigkeitsverteilung der Molekeln durch das Temperatur- und Konzentrationsgefälle berücksichtigt. Mathematisch geht man in der Weise vor, daß die Entwicklungsfunktionen eines linearen Störansatzes aus der Boltzmannschen Fundamentalgleichung bestimmt werden. In der Fundamentalgleichung wird die Bewegung der Molekeln bekanntlich in eine freie Phase und eine Stoßphase unterteilt ${ }^{10}$. Die Molekeln werden damit als „quasi-ideal“ angesehen, indem ihr Wechselwirkungspotential nur die Anisotropie der Richtungs- bzw. Geschwindigkeitsverteilung beim Stoß bestimmen soll ${ }^{16}$. Dies gilt auch für den Fall, daß neben abstoßenden Kräften anziehende (etwa van der Waals-Kräfte) berücksichtigt werden, z. B. durch einen Kraftansatz der Form ${ }^{17}$

$$
F=K / r^{9}-K^{\prime} / r^{5}
$$

( $r=$ Molekelabstand, $K$ und $K^{\prime}=$ Konstanten).

Die Massenabhängigkeit der Geschwindigkeitsanisotropie bedingt einen bevorzugten Transport einer Komponente in Richtung des Temperaturgefälles, der mit einem bevorzugten Impulstransport in dieser Richtung verknüpft ist. Wegen der aus mechanischen Gründen zu fordernden Gleichheit des $\mathrm{Ge}$ samtdruckes in allen Teilen des verschieden temperierten Gefäßes transportiert die andere Komponente bevorzugt Impuls in der entgegengesetzten Richtung.

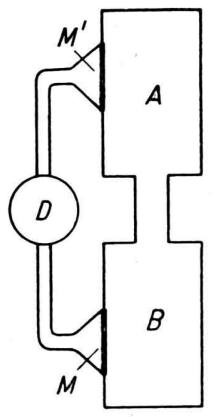

Abb.5. Grundprinzip der Anordnung zur Nachprüfung der Gültigkeit von Gl. (4) für ein im Temperaturgefälle befindliches ideales Gasgemisch.

Im stationären Zustand liegt eine bestimmte Entmischung der Gase vor, die dadurch gekennzeichnet ist, daß der von der Komponente i zum Druck auf die Wand gelieferte Beitrag $p_{\mathrm{i}}$ in den verschieden temperierten Teilen des Gefäßes verschieden und mit dem Gesamtdruck $p_{0}$ durch den Molenbruch $\gamma$ verknüpft ist. Für zwei Raumpunkte mit den Temperaturen $T$ und $T^{\prime}$ gilt also

$$
\begin{gathered}
p_{\mathrm{i}}=\gamma_{\mathrm{i}} p_{0}, \\
p_{\mathrm{i}}^{\prime}=\gamma_{\mathrm{i}}^{\prime} p_{0},
\end{gathered}
$$

d. h. für die Entmischung im Sinne von Gl. (1)

$$
\Delta \gamma=\gamma_{\mathrm{i}}{ }^{\prime}-\gamma_{\mathrm{i}}=\frac{p_{\mathrm{i}}{ }^{\prime}-p_{\mathrm{i}}}{p_{0}} .
$$

Wegen der Bedeutung von Gl. (4) für die Abgrenzung des Realitätseinflusses soll ihre Gültigkeit für ein als ideal anzusehendes, im Temperaturgefälle be-

16 Die Bezeichnung ,ideal“ gilt im folgenden immer im Sinne von „quasi-ideal“.

17 R. C. J on e s, Physic. Rev. 59, 1019 [1941]. 
findliches Gasgemisch noch experimentell geprüft werden.

Das Grundprinzip der dazu verwendeten Anordnung ist in Abb. 5 dargestellt. Das Teilgefäß A einer Thermodiffusionsapparatur, das die höhere absolute Temperatur $T^{\prime}$ besitzen möge, ist über die semipermeable Membrane $\mathbf{M}^{\prime}$, das Differentialmanometer $D$ und die semipermeable Membrane $M$ mit dem Teilgefäß B der Temperatur $T$ verbunden. A und B sind mit einem binären Gasgemisch gefüllt, von dem

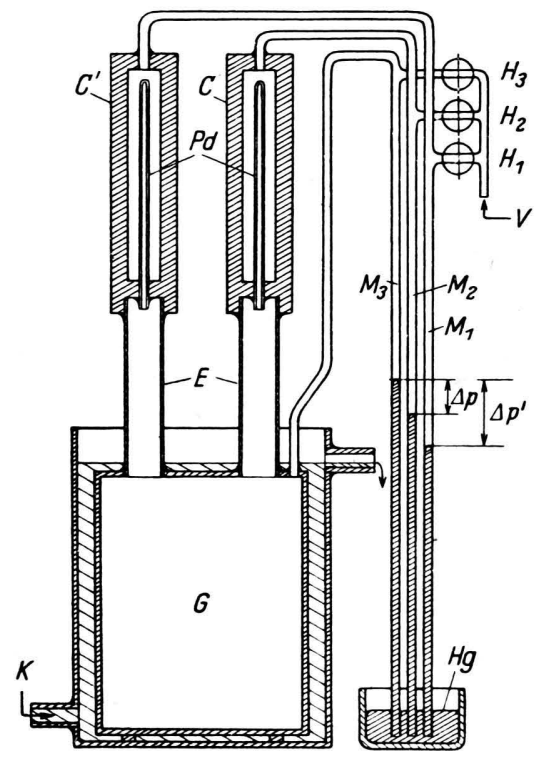

Abb. 6. Ausführung der in Abb. 5 im Prinzip dargestellten Anordnung. $\mathrm{M}_{3}$ zeigt die Stellung des Quecksilbers vor, $\mathrm{M}_{2}$ und $\mathrm{M}_{1}$ nach dem Einfüllen des Argons $(\mathrm{V}=$ Vakuumanschluß; $\mathrm{K}=$ Kühlwasser).

nur eine Komponente die Membranen passieren kann. Der Temperaturgradient innerhalb der Membranen möge gleich Null sein, so daß keine Störungen durch Thermoosmose ${ }^{18}$ auftreten können.

Nach Gl. (4) und Gl. (1) hat man bei einem idealen Gasgemisch einen Ausschlag $\Delta p$ an $\mathrm{D}$ zu erwarten:

$$
\Delta p=p_{0} \bar{\gamma}(\overline{1}-\bar{\gamma}) \alpha_{\text {ideal }} \ln T^{\prime} / T .
$$

Bei der Durchführung des Versuches ${ }^{19}$ wurde ein Gemisch aus $43 \%$ Wasserstoff und 57\% Argon unter einem Gesamtdruck von 576 Torr verwendet. Als semipermeable

18 Vgl. z. B. K. Wirtz, Z. Naturforschg. 3a, 380 [1948].

19 Ein ähnlicher Versuch, allerdings mit anderer Zielsetzung, ist von Zen-ichi Shibata u. Hiroshi $\mathrm{K}$ i t a g a w a (J. Fac. Sci., Hokkaido Imp. Univ., Ser. III, Chem. Vol. II, 223) mit einem $\mathrm{H}_{2} / \mathrm{H}_{2} \mathrm{O}$-Gemisch ausgeführt worden.

\begin{tabular}{|c|c|c|c|c|c|}
\hline $\begin{array}{c}\text { Vers.- } \\
\text { Nr. }\end{array}$ & $\begin{array}{c}T^{\prime} \\
{\left[{ }^{0} \mathrm{abs}\right]}\end{array}$ & $\begin{array}{c}T \\
{\left[{ }^{0} \mathrm{abs}\right]}\end{array}$ & $\begin{array}{c}\Delta p^{\prime} \\
\text { [Torr] }\end{array}$ & $\begin{array}{c}\Delta p \\
\text { [Torr] }\end{array}$ & $\begin{array}{c}\Delta p^{\prime}-\Delta p \\
\text { [Torr] }\end{array}$ \\
\hline 1 & 722 & 637 & 41,5 & 35,0 & 6,5 \\
2 & 687 & 582 & 37,0 & 28,5 & 8,5 \\
3 & 659 & 468 & 36,5 & 19,6 & 16,9 \\
4 & 617 & 521 & 33,0 & 24,5 & 8,5 \\
\hline
\end{tabular}

Tab. 2. Ergebnis der Versuche, die mit der in Abb. 6 dargestellten Apparatur ausgeführt wurden. Gesamtdruck in G: 576 Torr. Molenbruch des Wasserstoffs in G: 0,43; Temperatur in $\mathrm{G}: T_{\mathrm{n}}=290^{\circ}$ abs.

Membranen dienten geheizte Palladiumrohre. Der Aufbau der Apparatur geht aus Abb. 6 hervor: Die Palladiumrohre Pd sind in von außen elektrisch geheizten Kupferzylindern $\mathrm{C}$ und $\mathrm{C}^{\prime}$ untergebracht, deren Temperatur mit Thermoelementen bestimmt werden kann. Der Innenraum von Pd steht über dünnwandige Eisenrohre $\mathrm{E}$ mit einem durch Wasser gekühlten Gasvorrat G in Verbindung (Kühlwassertemperatur $T_{0}=290^{\circ}$ abs). An den Innenraum von $\mathrm{C}$ und $\mathrm{C}^{\prime}$ und an den Gasvorrat $\mathrm{G}$ sind Manometer $\mathrm{M}_{1-3}$ angeschlossen. Die einzelnen Räume können über die Hähne $\mathrm{H}_{1-3}$ evakuiert bzw. mit Gas gefüllt werden.

$\mathrm{C}$ und $\mathrm{C}^{\prime}$ werden auf die Temperaturen $T$ und $T^{\prime}$ eingestellt, die beide genügend hoch liegen, um das Palladium durchlässig für Wasserstoff zu machen. Nach vollständigem Leerpumpen aller Räume bei geöffneten Hähnen $\mathrm{H}_{1-3}$ werden $\mathrm{H}_{1}$ und $\mathrm{H}_{2}$ geschlossen. Danach wird über $\mathrm{H}_{3}$ in $\mathrm{G}$ zunächst reiner Wasserstoff eingelassen (etwa 300 Torr). Wegen der Durchlässigkeit der Palladiumrohre für Wasserstoff muß sich nach einiger Zeit in $C$ und $C^{\prime}$ der gleiche Druck wie in $\mathrm{G}$ einstellen, obwohl $\mathrm{C}$ und $\mathrm{C}^{\prime}$ verschiedene Temperaturen besitzen. Der endgültige Wasserstoffdruck in allen Teilen der Apparatur sei $p$. Nun wird das Gefäß G bis zum Enddruck $p_{0}$ mit Argon aufgefüllt. Sobald sich die Gase genügend vermischt haben, steigt der Druck in $\mathrm{C}$ und $\mathrm{C}^{\prime}$ um $\Delta p$ bzw. $\Delta p^{\prime}$ gegenüber dem Ausgangsdruck $p$ an.

In Tab. 2 sind die Ergebnisse von 4 Versuchen zusammengestellt, die mit verschiedenen Werten von $T$ und $T^{\prime}$ durchgeführt wurden ${ }^{20}$. Aus jedem Versuch läßt sich 3-mal der Thermodiffusionsfaktor gewinnen, entsprechend

$$
\begin{aligned}
& \alpha=\frac{\Delta p}{p_{0} \bar{\gamma}(1-\bar{\gamma}) \ln T / T_{0}}, \text { für } \bar{T}=\frac{1}{2}\left(T+T_{0}\right) ; \\
& \alpha=\frac{\Delta p^{\prime}}{p_{0} \bar{\gamma}(1-\bar{\gamma}) \ln T^{\prime} / T_{0}}, \text { für } \bar{T}=\frac{1}{2}\left(T^{\prime}+T_{0}\right) ; \\
& \alpha=\frac{\Delta p^{\prime}-\Delta p}{p_{0} \bar{\gamma}(1-\bar{\gamma}) \ln T^{\prime} / T}, \text { für } \bar{T}=\frac{1}{2}\left(T^{\prime}+T\right) .
\end{aligned}
$$

20 Die geringfügige Änderung des Wasserstoffdruckes in $\mathrm{G}$ auf Grund des Durchtretens von $\mathrm{H}_{2}$ durch die PdRohre nach dem Einfüllen des Argons ist in den $\Delta p^{\prime}$ bzw. $\Delta p$-Werten der Tab. 2 bereits berücksichtigt. 
In Abb. 7 sind alle mit den Gl. $(6 a-c)$ berechneten Thermodiffusionsfaktoren in Abhängigkeit von der mittleren absoluten Temperatur $\bar{T}$ aufgetragen. Man erkennt, daß die Meßpunkte ziemlich gut auf einer Geraden liegen, die zu höheren mittleren Temperaturen ansteigt.

Um die Gültigkeit der Gl. (4) für ein im Temperaturgefälle befindliches ideales Gasgemisch nachzuweisen, müssen unsere durch Druckmessung gewonnenen $\alpha$-Werte mit denen verglichen werden, die durch Bestimmung der Entmischung im Temperaturfeld erhalten wurden. Aus einer Untersuchung von I b b s , $\mathrm{Grew}$ und $\mathrm{Hirst}{ }^{21}$, die bei einer mittleren Tem-

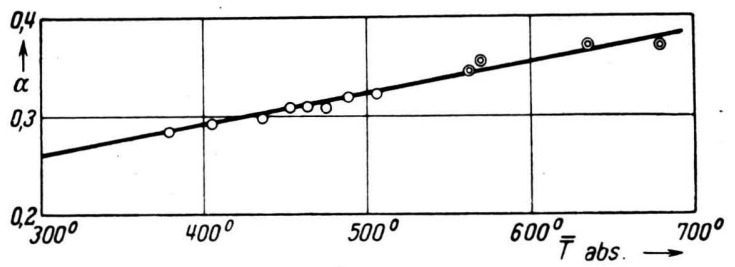

Abb. 7. Thermodiffusionsfaktor $\alpha$ eines aus $43 \% \mathrm{H}_{2}$ und $57 \%$ A bestehenden Gemisches unter einem Gesamtdruck von 576 Torr in Abhängigkeit von der mittleren Temperatur $\bar{T}$, bestimmt mit der in Abb. 6 dargestellten Apparatur unter Verwendung der Gl. (6a) und (6 b) (Kreise), sowie der Gl. (6c) (Doppelkreise).

peratur von etwa $260^{\circ}$ durchgeführt wurde, entnehmen wir für $\mathrm{H}_{2} / \mathrm{A}$

$$
a_{\left(55,6 \% \mathrm{H}_{2}\right)}=0,288 ; \quad a_{\left(47 \% \mathrm{H}_{2}\right)}=0,255 .
$$

Daraus folgt durch lineare Extrapolation auf die von uns angewendete Wasserstoffkonzentration von $43 \%$

$$
\alpha=0,24_{0} .
$$

Verlängert man die in Abb. 7 dargestellte Gerade bis $\bar{T}=260^{\circ}$, so erhält man zum Vergleich

$$
\alpha=0,24_{5}
$$

in ausgezeichneter Übereinstimmung mit dem aus den Versuchen von Ibbs, Grew und Hirst berechneten Wert, womit die Gültigkeit von Gl. (4) für ein ideales Gasgemisch bestätigt sein dürfte.

\section{Der Realitätsanteil \\ der thermischen Entmischung}

Wir fragen nun, welche neuen Erscheinungen prinzipiell zu erwarten sind, wenn eine der Komponenten merklich real ist. Wir betrachten dazu das folgende

21 T. L. I b b s, K. E. Grew u. A. Hirst, Proc. physic. Soc. 41, 436 [1929]. hypothetische Gemisch: Die Komponente 1 allein möge das ideale Gasgesetz befolgen, die Komponente 2 allein die vereinfachte van der Waalssche Zustandsgleichung

$$
p_{2}+\mathrm{a} / V_{2}{ }^{2}=R T / V_{2} .
$$

Im Gemisch mögen beide Komponenten nur mit sich selbst zusammenstoßen. Jegliche Wechselwirkung von Molekülen der Sorte 1 mit solchen der Sorte 2 sei ausgeschlossen.

Das beschriebene Gemisch kann keine EnskogChapmansche Thermodiffusion zeigen, da dieser Effekt durch den Zusammenstoß verschiedenartiger Teilchen hervorgerufen wird. Jede Komponente wird sich vielmehr im Temperaturgefälle so verteilen, daß ihr Beitrag $p$ zum Druck auf die Wand im gesamten Gefäß konstant ist. Das Gemisch wird daher auch keinen Ausschlag des Differentialmanometers D der in Abb. 5 dargestellten Apparatur bewirken. (Wegen der Realität von 2 gilt jetzt nicht mehr die Beziehung 3.) Auf Grund des idealen Gasgesetzes folgt dann für die Komponente $1:^{22}(N=$ Zahl der Teilchen in der Volumeneinheit)

$$
T N_{1}=\dot{T}^{\prime} N_{1}{ }^{\prime},
$$

während sich für das Gas 2 nach Gl. (7) ergibt: $\left(N_{\mathrm{L}}=\right.$ Loschmidtsche Zahl)

$$
T N_{2}-\frac{N_{2}^{2} \mathrm{a}}{N_{\mathrm{L}} R}=T^{\prime} N_{2}{ }^{\prime}-\frac{N_{2}{ }^{2} \mathrm{a}}{N_{\mathrm{L}} R} .
$$

Setzt man z. B. $N_{1}=N_{2}$, so folgt durch Vergleich von (8) und (9): $N_{1}^{\prime} \neq N_{2}^{\prime}$, also eine thermische Entmischung der Gase, die mit der Enskog-Chapmanschen Thermodiffusion deshalb nichts zu tun haben kann, weil sie nur durch die Wechselwirkung gleichartiger Teilchen zustande gekommen ist. Wie man durch Vergleich von (8) und (9) weiter erkennt, verschwindet die Entmischung, wenn $\mathrm{a}=0$ ist bzw. wenn $N$ sehr klein oder $T$ sehr groß wird, d. h. allgemein wenn auch das Gas 2 als ideal angesehen werden kann.

Durch Kombination der Gl. (8) und (9) läßt sich die auf Grund der Realität der Komponente 2 zu erwartende Entmischung berechnen. Bei Einführung des Molenbruches werden die Formeln jedoch so unhand-

22 Die Verwendung der für ein isothermes System aufgestellten Zustandsgleichung zur Berechnung der Dichteverteilung eines in einem Temperaturgefälle befindlichen Gases ist natürlich nur gerechtfertigt, solange die Temperaturänderung auf eine freie Weglänge klein ist gegen die mittlere Temperatur der betrachteten Stelle. Diese Voraussetzung ist aber bei den $\mathrm{zu}$ interpretierenden Versuchen stets erfüllt. 
lich, daß wir einen etwas anderen Weg einschlagen wollen, der außerdem physikalisch übersichtlicher sein dürfte. Dazu schreiben wir die Gln. (3a) und (3b) in der für das neue Gemisch gültigen Form an. $p_{0}$ möge wieder der Gesamtdruck des Gemisches sein, während wir mit $p_{0 \text { ideal }}$ den Druck bezeichnen wollen, den das Gemisch ausüben würde, wenn beide Komponenten ideal wären. Es gilt dann

$$
p_{0 \text { ideal }}=p_{0}+\mathrm{a} / V_{2}{ }^{2} .
$$

Die zu Gl. (3 a) analoge Beziehung lautet infolgedessen, da das Gas 1 ideal ist,

$$
p_{1}=\gamma_{1} p_{0 \text { ideal }}=\gamma_{1}\left(p_{0}+\mathrm{a} / V_{2}{ }^{2}\right),
$$

während man statt Gl. (3b) zu schreiben hat

$$
p_{1}{ }^{\prime}=\gamma_{1}{ }^{\prime} p_{0}{ }^{\prime} \text { ideal }=\gamma_{1}{ }^{\prime}\left(p_{0}+\mathrm{a} / V_{2}{ }^{2}\right) \text {. }
$$

Die Anschlußbedingung zwischen heißem und kaltem Gefäß (s. oben)

liefert dann

$$
p_{1}=p_{1}{ }^{\prime}
$$

$$
\gamma_{1}^{\prime}=\gamma_{1}\left(p_{0}+\mathrm{a} / V_{2}{ }^{2}\right) /\left(p_{0}+\mathrm{a} / V_{2}{ }^{\prime 2}\right),
$$

wodurch sich für die gesuchte Entmischung ergibt:

$$
\gamma_{1}{ }^{\prime}-\gamma_{1}=\bar{\gamma}_{1}\left[\left(p_{0}+\mathrm{a} / V_{2}{ }^{2}\right) /\left(p_{0}+\mathrm{a} /{V_{2}}{ }^{2}\right)-1\right] .
$$

Dabei wurde noch $\gamma_{1}$ auf der rechten Seite durch den mittleren Molenbruch $\bar{\gamma}_{1}$ ersetzt. Da bei endlicher Temperaturdifferenz sicher $V_{2} \neq V_{2}^{\prime}$ ist, folgt nach Gl. (15) wieder, daß die Entmischung nur dann verschwindet, wenn $\mathrm{a} / \mathrm{V}_{2}{ }^{2}=\mathrm{a} / \mathrm{V}_{2}^{\prime}{ }^{2}=0$ ist, wenn also auch die zweite Komponente dem idealen Gasgesetz gehorcht.

Die Molvolumina $V_{2}$ und $V_{2}^{\prime}$ können im Prinzip mit Gl. (7) berechnet werden, da eine Wechselwirkung zwischen den Gasen 1 und 2 ausgeschlossen wurde. Für den Zweck einer Abschätzung des Effektes genügt es jedoch, $V_{2}$ und $V_{2}^{\prime}$ über das ideale Gasgesetz einzuführen, wodurch sich die endgültige Formel ergibt:

mit

$$
\gamma_{1}{ }^{\prime}-\gamma_{1}=\bar{\gamma}_{1}\left[\left(1+\beta / T^{2}\right) /\left(1+\beta / T^{\prime 2}\right)-1\right]
$$

$$
\beta=\frac{\mathrm{a}}{R^{2}}\left(1-\bar{\gamma}_{1}\right)^{2} p_{0} .
$$

Bei $T^{\prime}>T$ ist $\gamma_{1}^{\prime}-\gamma_{1}$ positiv. Die ideale Komponente reichert sich also im Gebiet höherer Temperatur an, wobei die Größe der Entmischung etwa linear mit dem Gesamtdruck zunimmt.

Gl. (16) wurde unter der Voraussetzung abgeleitet, daß die eine Komponente völlig ideal sei, und daß keine Wechselwirkungen zwischen den beiden Gaspartnern auftreten. Wir erweitern jetzt unsere Betrachtung durch Berücksichtigung der Zusammenstöße ungleicher Teilchen, nehmen jedoch die eine Komponente weiterhin als ideal an.

Der zusätzliche Effekt kann wegen der Idealität des einen Partners nur in der Enskog-Chapmanschen Thermodiffusion bestehen. Da die eine Entmischungserscheinung durch die Wechselwirkung gleichartiger, die andere durch die ungleichartiger Moleküle zustandekommt, können beide als in erster Näherung von einander unabhängig angesehen werden. Die zugeordneten Entmischungen $\Delta \gamma$ werden sich also bei $\Delta \gamma \ll 1$ nahezu additiv verhalten:

$$
\Delta \gamma \approx \Delta \gamma_{\text {ideal }}+\Delta \gamma_{\text {real }} .
$$

Der erste Term auf der rechten Seite ergibt sich auf Grund von Gl. (1) mit $\alpha=\alpha_{\text {ideal }}$. Für den zweiten stellen wir eine zu (1) analoge Beziehung auf mit $\alpha=\alpha_{\text {real }}$. Sie liefert durch Vergleich mit (16)

$$
\alpha_{\text {real }}=\frac{1}{\left(1-\bar{\gamma}_{1}\right) \ln T^{\prime} / T}\left[\left(1+\beta / T^{2}\right) /\left(1+\beta / T^{\prime 2}\right)-1\right] .
$$

Beschreibt man schließlich auch die Gesamtentmischung durch Gl. (1), so folgt auf Grund von (18):

$$
\alpha \approx \alpha_{\text {ideal }}+\alpha_{\text {real }} .
$$

$\mathrm{Da} \beta \alpha_{\text {real }}$ und $\alpha_{\text {ideal }}$ nebeneinander eine in erster Näherung selbständige physikalische Bedeutung besitzen, geht einmal aus dem Hinweis hervor, daß die eine Größe wesentlich durch die Wechselwirkung gleichartiger, die andere durch die Wechselwirkung verschiedenartiger Molekeln bestimmt ist. Zum anderen aus der Tatsache, daß sich ihre Zahlenwerte im Prinzip mit der in Abb. 5 skizzierten Apparatur einzeln bestimmen lassen. Füllt man nämlich das Thermodiffusionsgefäß mit dem zuletzt diskutierten Gemisch aus einer realen und einer idealen Komponente, von denen nur eine die Membranen passieren kann, so wird durch Beobachtung der Druckdifferenz an D nach Gl. (5) $a_{\text {ideal }}$ zu gewinnen sein, während sich durch Messung der Entmischung zwischen A und B der Wert von $\alpha$ und damit nach Gl. (20) auch der von $\alpha_{\text {real }}$ ergibt ${ }^{23}$.

${ }^{23}$ Um die Trennung von $\alpha_{\text {real }}$ und $\alpha_{\text {ideal }}$ experimentell durchzuführen, wird man die Pd-Rohre zweckmäßig durch Membranen ersetzen, die schon bei Zimmertemperatur semipermeabel sind (etwa Kautschuk für $\mathrm{NH}_{3} / \mathrm{He}-\mathrm{Ge}-$ mische), da der Realitätseffekt mit steigender Temperatur schnell kleiner wird [vgl. Abb. 3 bzw. Gl. (16)]. 


\section{Vergleich \\ zwischen Theorie und Experiment}

Von den im ersten Teil der Arbeit experimentell untersuchten Gaspaaren entspricht das System $\mathrm{H}_{2} / \mathrm{CO}_{2}$ am besten den der Rechnung zugrundegelegten Voraussetzungen, da Wasserstoff auch bei relativ hohen Drucken noch als hinreichend ideal angesehen werden kann. Wir werden daher das Ergebnis von Theorie und Experiment an diesem Gaspaar vergleichen. Nach der Enskog-Chapmanschen Theorie nehmen wir $\alpha_{\text {ideal }}$ als im wesentlichen druckunabhängig an und gewinnen seinen Wert durch Extrapolation der in Abb. 2 dargestellten experimentellen Kurve auf den Druck 0. Mit der van der Waals-Konstante ${ }^{24}$ des $\mathrm{CO}_{2}$ $\mathrm{a}=6,3 \mathrm{~atm} l^{2} \mathrm{~mol}^{-2}$ ergibt sich dann nach (19) und (20) für die Druckabhängigkeit von $\alpha$ der in Abb. 2 gestrichelt eingezeichnete Verlauf. Die Übereinstimmung zwischen Theorie und Experiment kann im Hinblick auf die in der Theorie enthaltenen Vereinfachungen als durchaus befriedigend angesehen werden. Auch die negative Temperaturabhängigkeit von $\alpha$ bei hohen Drucken und die besondere Form der Konzentrationsabhängigkeit werden durch die Gln. (19) und (20) qualitativ richtig wiedergegeben, wie aus den gestrichelten Kurven der Abb. 3 und 4 hervorgeht.

Für andere Gaspaare lassen sich nach Gln. (19) und (20) einige qualitative Voraussagen machen: So kann man erwarten, daß die Druckabhängigkeit von $\alpha$ klein sein wird, wenn beide Komponenten weitgehend ideal sind, wie dies z. B. bei $\mathrm{H}_{2} / \mathrm{N}_{2}$ der Fall ist. Aus Abb. 2 geht hervor, daß dieses Gaspaar tatsächlich nur eine sehr schwache Druckabhängigkeit zeigt. Für das Vorzeichen der Druckabhängigkeit läßt sich weiter die Regel aufstellen, daß bei normalem Vorzeichen von $\alpha_{\text {ideal }}$ (die schwere Komponente reichert sich an der kalten Wand an) positive Druckabhängigkeit dann $\mathrm{zu}$ erwarten ist, wenn die schwerere Komponente die größere Attraktionskonstante besitzt. Dies ist bei allen in der Abb. 2 verzeichneten Gaspaaren der Fall, außer beim $\mathrm{N}_{2} / \mathrm{CH}_{4}$, das auch tatsächlich eine negative Druckabhängigkeit aufweist, die bei höheren Drucken wahrscheinlich zu einem Vorzeichenwechsel von $\alpha$ führt.

Ein solcher Vorzeichenwechsel auf Grund des Einflusses der Realität kann einwandfrei schon bei niedrigen Drucken am System $\mathrm{N}_{2} / \mathrm{NH}_{3}$ nachgewiesen wer-

24 Es wurde die aus $p v$-Messungen abgeleitete van der Waals-Konstante verwendet. Vgl. A. E u c k e n, Lehrbuch der Chemischen Physik $\mathrm{II}_{1}$, 2. Auflage, S. 228, Leipzig 1943. den, bei dem ebenfalls die leichtere Komponente das größere van der Waalssche a besitzt. Da dieses $\mathrm{Ge}$ paar jedoch nur eine sehr kleine thermische Entmischung zeigt, läßt sich $\alpha$ mit der in Abb. 1 dargestellten Apparatur nur schwierig bestimmen. Das Gemisch wurde daher mit einem Clusius-Dickelschen Trennrohr untersucht. Bei einer mittleren Temperatur von $50^{\circ} \mathrm{C}$ und äquimolarer Zusammensetzung ergibt sich unterhalb von 1,8 atm eine Anreicherung des $\mathrm{NH}_{3}$ am oberen, oberhalb dieses Druckes eine Anreicherung am unteren Rohrende. Daraus folgt unabhängig von den Vervielfachungseigenschaften des Trennrohres, daß der Thermodiffusionsfaktor des Gemisches bei $\approx 1,8 \mathrm{~atm}$ sein Vorzeichen wechselt.

Aus dem letzten Beispiel ist zu ersehen, daß der Realitätseinfluß für Gase mit kleinem $\alpha_{\text {ideal }}$ schon bei relativ niedrigen Drucken von wesentlicher Bedeutung sein kann. Es scheint, daß auch der von Gr e w ${ }^{25}$ mit dem Trennrohr beobachtete Vorzeichenwechsel der Thermodiffusion von $\mathrm{Ne} / \mathrm{NH}_{3}$ bei $\approx 1$ atm Gesamtdruck und $25 \% \mathrm{NH}_{3}$ Gehalt mehr durch den Realitätseffekt bestimmt ist als durch den Unterschied im Durchmesser der Moleküle, den Grew allein für die Erklärung des Phänomens heranzieht.

Abschließend kann festgestellt werden, daß die wesentlichen Züge der experimentellen Ergebnisse bereits durch die hier entwickelte, stark vereinfachte Theorie des Realitätseffektes richtig wiedergegeben werden. Eine vollständige theoretische Beschreibung wird natürlich nur mit einer Zustandsgleichung gelingen, die alle Möglichkeiten der Wechselwirkung sowie den Einfluß des Eigenvolumens der Moleküle berücksichtigt.

$$
\begin{gathered}
\text { Anhang: } \\
\text { Der Diffusions - Thermoeffekt } \\
\text { bei realen Gasen }
\end{gathered}
$$

Wir haben schon früher darauf hingewiesen ${ }^{11}$, daß es zu dem hier beschriebenen, auf der Realität der Gase beruhenden Entmischungsanteil einen Umkehreffekt geben muß, der darin besteht, daß sich beim Ineinanderdiffundieren verschieden stark realer Gase im Gas vorübergehend ein Temperaturgefälle aufbaut. Kinetisch kann man diesen Effekt leicht einsehen am Beispiel des oben diskutierten Gemisches aus der idealen Komponente 1 und der realen Komponente 2 , in dem jegliche Wechselwirkung zwischen 1 und 2 ausgeschlossen ist. Bei einem Diffusionsprozeß wird sich der mittlere Abstand der realen Moleküle 2 in der an 2 ärmeren Gefäßhälften ver-

25 K. E. Grew, Nature [London] 150, 320 [1942]. 
mindern, in der an 2 reicheren dagegen vergrößern. In der ersteren wird dabei wegen des Überganges von potentieller in kinetische Energie vorübergehend eine Erwärmung, in der zweiten dagegen eine Abkühlung zu verzeichnen sein. Bei einem wirklichen Gasgemisch tritt infolge des Zusammenstoßes ungleichartiger Teilchen zusätzlich der Umkehreffekt der EnskogChapmanschen Thermodiffusion auf ${ }^{26}$.

In der Zwischenzeit ist eine Arbeit von L. W aldmann erschienen ${ }^{27}$, die sich mit der Druck- und Temperaturabhängigkeit der Wärmeerscheinungen bei der Diffusion beschäftigt. Darin wird theoretisch von den Realitätseffekten jedoch nur derjenige Term berücksichtigt, der zu einer Änderung der mittleren

26 Den letzteren pflegt man gewöhnlich allein als Diffusionsthermoeffekt zu bezeichnen. Er wurde erstmals von K. Clusius u. L. Wa l d m a n $\mathrm{n}$ beobachtet (Naturwiss. 30, 711 [1942]). Vgl. auch L. W a l d m a n n, Z. Naturforschg. 1, 59 [1946].

27 L. Wa l d m a n n, Z. Naturforschg. 4 a, 105 [1949].

28 Auf diesen Tatbestand machte auch R. H a a s e aufmerksam (Z. Physik 127, S. 5, Anm. 2 [1950].
Temperatur des Gesamtsystems führt. Waldmann muß daher in seinem Strömungsapparat eine für beide Meßzellen in erster Näherung gleichmäßige Änderung der Temperatur mit dem Druck erwarten. Tatsächlich wird jedoch ein Aufspreizen der TemperaturDruck-Kurven beobachtet, für die eine Erklärung deshalb nicht gegeben werden kann, weil der Thermodiffusionsfaktor für den Untersuchungsbereich als druckunabhängig angenommen wird. Aus unseren Messungen und theoretischen Überlegungen geht jedoch hervor, daß diese Annahme für Gaspaare mit kleinem $\alpha_{\text {ideal }}$ und großem Unterschied der van der Waals-Konstanten auch bei Drucken unter einer Atmosphäre nicht berechtigt ist, vor allem, wenn bei tieferen Temperaturen gemessen wird ${ }^{28}$. Das Aufspreizen der Kurven dürfte also im wesentlichen auf dem oben postulierten Umkehreffekt des Realanteils der thermischen Entmischung beruhen.

Hrn. Prof. Dr. W. Wa lcher danke ich für sein Interesse an dieser Arbeit und für mehrere aufschlußreiche Diskussionen.

\section{NOTIZEN}

\section{Erhebungswinkelschwankungen bei der Kurzwellenausbreitung \\ Von W a l ter B ud d e*}

(Z. Naturforschg. 5 a, 465 [1950]; eingeg. am 12. August 1950)

Zum Zwecke der Bestimmung der Grenzen der Richtungsmessung mit Raumwellen wurden Untersuchungen über die Großkreisausbrechungen von Kurzwellen durchgeführt ${ }^{1}$. Die verwendete Anlage gestattete die gleichzeitige Bestimmung des Ausstrahlungserhebungswinkels. Aus Messungen dẹs Erhebungswinkels lassen sich weitgehende Rückschlüsse auf den Ausbreitungsmechanismus ziehen 2 .

Infolge der Unruhe in der Ionosphäre wird man ein Schwanken des Erhebungswinkels um einen bestimmten Mittelwert erwarten. Bei der Übertragung von München nach Koelby (Dänemark) wurden für eine Frequenz von $9830 \mathrm{kHz}$ kurzzeitige Schwankungen des Ausstrahlungserhebungswinkels festgestellt. Die Dauer dieser Schwankungen war durchschnittlich einige Minuten, ihre Größe betrug bis $\mathrm{zu} \pm 4^{\circ}$. Außerdem wurde an verschiedenen

1 W. Cron e, Möglichkeiten und Grenzen der Richtungsmessung mit Raumwellen, Bericht des FerdinandBraun-Institutes Landsberg a. Lech [1944]; W. B u d d e, Diss. T. H. München 1947, Z. Naturforschg. 5a, 291 [1950].

2 W. Crone, K. Krüger, G. Goubeau u. J. Z e nneck, Hochfrequenztechnik u. Elektroakustik 48, 1 [1936]; L. H a m berger u. K. R a wer, Z. Naturforschg. 2 a, 521 [1947].

* Aachen, Karl-Marx-Allee 154.
Tagen eine stetige Veränderung des Erhebungswinkels im Verlauf mehrerer Stunden beobachtet, und zwar an manchen Tagen zu größeren Werten hin, an anderen Tagen zu kleineren Werten. Auch sprunghafte Änderungen des Ausstrahlungserhebungswinkels wurden gemessen und lassen auf gelegentliche plötzliche Änderungen des Übertragungsweges schließen. Die Messungen wurden nur am Tage durchgeführt.

\section{Reversible Veränderungen in der Randschicht von Selengleichrichtern}

Von A. Hof f mann, F. Rose, E. Waldköt ter

Dienststelle Pretzfeld der Siemens-Schuckert-Werke

$$
\text { und von E. Nits che }
$$

Gerätewerk der Siemens-Schuckert-Werke Berlin

(Z. Naturforschg. 5 a, 465-467 [1950]; eingeg. am 3. August 1950)

Bei den Untersuchungen von Selengleichrichtern sind zwei verschiedene Erscheinungen aufgefallen, die sich wahrscheinlich auf ein und dieselbe Ursache zurückführen lassen.

Es handelt sich einmal um eine Erhöhung des Durchlaßwiderstandes, die sich an manchen Objekten mehr oder weniger stark bemerkbar macht, wenn man von einer allein in Durchlaßrichtung wirksamen Belastung zu der betriebsmäßigen Belastung übergeht, in der der Gleichrichter während eines Teils der Periode in Sperrichtung beansprucht wird. Der „Durchlaßwiderstand bei reiner Flußbelastung“ ist kleiner als der „Durchlaßwiderstand 